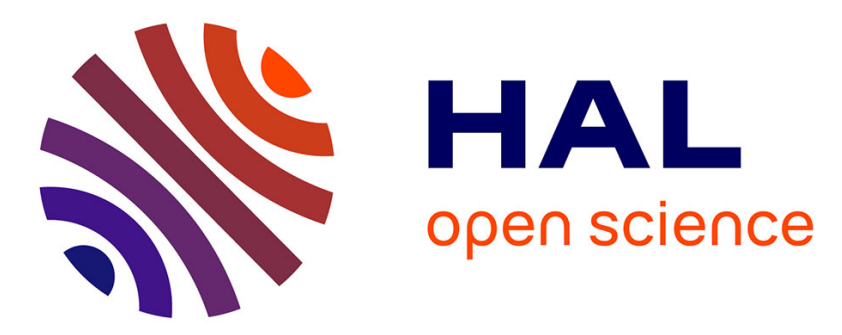

\title{
Insecticides systémiques: de nouveaux risques pour les insectes pollinisateurs
}

Roland Charvet, Mehrnaz Katouzian-Safadi, Marc-Edouard Colin, Patrice A Marchand, Jean-Marc Bonmatin

\section{- To cite this version:}

Roland Charvet, Mehrnaz Katouzian-Safadi, Marc-Edouard Colin, Patrice A Marchand, Jean-Marc Bonmatin. Insecticides systémiques: de nouveaux risques pour les insectes pollinisateurs. Annales Pharmaceutiques Françaises, 2004, 62 (1), pp.29-35. 10.1016/S0003-4509(04)94278-2 . hal-02082454

\section{HAL Id: hal-02082454 https://hal.science/hal-02082454}

Submitted on 29 Sep 2021

HAL is a multi-disciplinary open access archive for the deposit and dissemination of scientific research documents, whether they are published or not. The documents may come from teaching and research institutions in France or abroad, or from public or private research centers.
L'archive ouverte pluridisciplinaire HAL, est destinée au dépôt et à la diffusion de documents scientifiques de niveau recherche, publiés ou non, émanant des établissements d'enseignement et de recherche français ou étrangers, des laboratoires publics ou privés.

\section{(c)(1)}

Distributed under a Creative Commons Attribution| 4.0 International License 


\title{
Insecticides systémiques : de nouveaux risques pour les insectes pollinisateurs
}

\author{
R. Charvet (1), M. Katouzian-Safadi (2), M.-E. Colin (3), P.-A. Marchand (1), \\ J.-M. Bonmatin (1)
}

Résumé. Un nouveau type d'insecticide systémique, l'imidaclopride, est largement utilisé depuis 1994 en enrobage de semences. En effet, son mode d'application et son efficacité ont permis de réduire de manière très significative les quantités habituellement utilisées par pulvérisation. Néanmoins, suite à de fortes dépopulations de ruchers, I'insecticide imidaclopride est suspecté d'avoir des effets délétères sur les insectes pollinisateurs. De récentes études ont montré que cet insecticide présente une toxicité chronique et sub-létale pour des doses de l'ordre de la partie par milliard $(\mu \mathrm{g} / \mathrm{kg})$, ou moins, puisqu'on on observe un taux de $50 \%$ de mortalité chez l'abeille en dix jours pour une concentration de $0,1 \mu \mathrm{g} / \mathrm{kg}$ d'imidaclopride dans une nourriture contaminée. II s'est donc avéré nécessaire de pouvoir détecter de telles quantités d'imidaclopride dans les sols, les plantes, les fleurs et les pollens. Par de nouvelles méthodes analytiques, nous avons mis en évidence la bio-disponibilité de l'imidaclopride dans l'environnement et permis d'appréhender les risques encourus par les abeilles.

Mots-clés : Insecticide, Imidaclopride, Sols, Fleurs, Pollens, Abeilles.

\section{Problèmes environnementaux : pesticide}

Pour lutter contre les ravages des insectes sur les

(1) Centre de Biophysique Moléculaire, CNRS et Université Orléans, F45071 Orléans Cedex 02.

(2) Centre d'Histoire des Sciences et des Philosophies Arabes et Médiévales, CNRS, 7, rue Guy Môquet, BP 8, F94801 Villejuif Cedex.

(3) Laboratoire de Pathologie Comparée des Invertébrés, Université de Montpellier II, CC 101, Bâtiment 24, Place Eugène Bataillon, F34095 Montpellier Cedex 5.
Summary. Imidacloprid, a new systemic insecticide used as seed-dressing, has been widely used in France since 1994. Its application mode and its efficiency allow a significant reduction in comparison with the usual quantity of chemicals used during pulverising treatment. But the insecticide imidacloprid is suspected to have harmful effects on the pollinators as many bees have died since its introduction. Recent studies have shown that imidacloprid has chronic and sublethal toxicities at levels of $\mu \mathrm{g} / \mathrm{kg}$ or less. It was therefore necessary to detect imidacloprid at these levels in soils, plants, flowers, and pollens. With this aim, we characterised the bio-availability of imidacloprid in the environment using a new quantitative analytical method, as a basis for the evaluation of the risk for bees.

Key-words: Insecticide, Imidacloprid, Soils, Flowers, Pollens, Bees.

Systemic insecticides: new risk for pollinator insects. R. Charvet, M. Katouzian-Safadi, M.-E. Colin, P.-A. Marchand, J.-M. Bonmatin, Ann Pharm Fr 2004, 62: 29-35.

cultures, l'industrie chimique a mis au point de nombreux insecticides. Beaucoup se sont révélés efficaces mais ont engendré nombre de problèmes environnementaux à plus long terme. Plusieurs types d'insecticides ont été utilisés depuis ces 40 dernières années pour protéger les cultures, notamment contre les insectes suceurs et piqueurs. La plupart de ces insecticides sont appliqués par pulvérisations avec la nécessité, pour être efficace, d'être utilisés en grande quantité. Ici, seule une faible quantité sert à protéger les cultures, alors que le reste se disperse avec les vents. De plus, cette utilisation nécessite de nombreuses applications pour maintenir la protection tout au 
long de la croissance des cultures. Années après années, ces pulvérisations intensives conduisent à une pollution de l'air, des sols et des eaux par lessivage et infiltration dans les sols. À terme, la pollution des écosystèmes engendre une dégradation de la qualité des eaux potables, des déséquilibres de la flore et des intoxications ponctuelles ou chroniques de la faune.

De nombreux pesticides ont donc été interdits a posteriori et, face à ces pollutions, des recherches ont été menées pour trouver des molécules plus spécifiques des insectes ciblés, afin de limiter les impacts négatifs sur le reste de la faune. Ainsi, de nouveaux insecticides ont été commercialisés et s'annonçaient d'une plus grande efficacité. Tout d'abord, ils ont souvent été conçus pour avoir une plus grande affinité pour le système nerveux des insectes que pour celui des mammifères. De plus, ces insecticides peuvent être utilisés en enrobage de semences. Une dose prédéfinie d'insecticide est donc appliquée autour des semences pour les protéger. Durant la croissance, l'insecticide diffuse via la sève, dans toute la plante, en assurant ainsi une bonne protection. Les insecticides ayant ces propriétés de diffusion sont appelés systémiques. Cette technique permet donc, a priori, de réduire les risques de pollution puisque les quantités utilisées sont optimisées et que l'insecticide n'est pas dissipé audelà de l'environnement direct de la semence et de la plante.

\section{Imidaclopride}

L'imidaclopride est l'un de ces insecticides systémiques utilisés comme enrobage de semences. Il a pour cible les insectes ravageurs, incluant les sauterelles, pucerons, mouches, termites, coccinelles, insectes de l'herbe et du sol [1]. Il est commercialisé en France depuis 1994 et est largement répandu dans le monde entier, notamment pour les cultures du riz, des céréales, du maïs, du tournesol, des pommes de terre, des légumes ou pour certains arbres fruitiers. Selon le type de culture, l'imidaclopride est utilisé sous forme d'enrobage de semence $\left(\right.$ Gaucho ${ }^{\circledR}$ ) ou en pulvérisation $\left(\right.$ Confidor $\left.^{\circledR}\right)$. L'imidaclopride est un néo-nicotinoïde agissant sur le système nerveux central par le biais des récepteurs post-synaptiques.
Il cible les récepteurs nicotiniques de l'acétylcholine (nAChR) provoquant une accumulation de ce neuromédiateur [2-5]. Ceci conduit à des tremblements musculaires, à une incoordination motrice, à la paralysie et à la mort de l'insecte à brève échéance [6].

\section{Problématique}

Depuis la mise sur le marché de l'imidaclopride, les organisations apicoles ont rapidement observé de fortes dépopulations d'abeilles. En quelques années, près d'un quart des ruches françaises a disparu, entraînant une chute spectaculaire de la production de miel au plan national. Par ailleurs, les apiculteurs professionnels et amateurs ont observé des comportements anormaux chez leurs butineuses. Ces comportements ont souvent été suivis de la mort de leurs abeilles et/ou du dépérissement de leurs ruches. Par la suite, il a été constaté que les nouveaux problèmes apicoles allaient en grandissant avec l'utilisation croissante de l'imidaclopride, en termes de surface et de multiplicité des cultures traitées. S'est donc posée la question de l'existence d'un lien de cause à effet.

L'existence de ce genre de lien est toujours très délicate à aborder car il faut pouvoir relier la toxicité de la molécule suspectée avec sa présence effective dans le milieu naturel de l'espèce menacée. Ainsi, au moment des premières observations de mortalité, la quantité d'imidaclopride susceptible d'intoxiquer les abeilles était évaluée à une cinquantaine de $\mu \mathrm{g} / \mathrm{kg}$, quantité bien supérieure à celle potentiellement présente dans les pollens. De plus, les premières études montraient que la quantité d'imidaclopride dans la plante décroît pour atteindre, au moment de la floraison, une quantité jugée négligeable. Le contexte est d'autant plus complexe que l'abeille est depuis longtemps menacée par de nombreux parasites (comme le varroa), par des virus ou encore par d'autres pesticides. Il devient alors difficile de définir la responsabilité d'un de ces facteurs lors d'intoxications ou de phénomènes de fragilisation de l'abeille, la rendant encore plus vulnérable aux autres facteurs.

Une étude a été lancée dès 1998 par la France (ministères de l'Agriculture et de l'Environnement) avec le soutien financier de la Communauté 
européenne. Un volet a été mené en collaboration avec de nombreux organismes de recherche (CNRS, INRA, AFSSA notamment) pour aborder les problèmes de contamination des sols, des végétaux et des pollens. En parallèle, la toxicité de l'imidaclopride pour les abeilles a été étudiée en laboratoire et en conditions contrôlées, grâce à des ruches sous tunnels. Ces dernières études ont pour objectif de se rapprocher des conditions réelles et, pour avantage, un contrôle de l'intoxication et une observation précise des symptômes.

\section{Toxicité}

Sur le plan comportemental, on assiste à une intense activité de butinage des abeilles à la floraison du tournesol et du maïs. Les butineuses récoltent le nectar et le pollen des nombreuses petites fleurs composant la périphérie de la tête du tournesol, également appelée capitule en floraison. L'activité de butinage se fait de manière particulièrement organisée et méthodique, fleur par fleur, assurant ainsi des rendements de récolte élevés en matière de pollen et de nectar.

$\mathrm{Au}$ contraire, sur les parcelles de tournesol traitées par enrobage de semences $\left(\right.$ Gaucho $^{\circledR}$ ou son concurrent Régent ${ }^{\circledR}$ ), l'activité de butinage dévoile souvent des comportements anormaux. Le butinage n'est plus alors systématique et ordonné, mais les abeilles présentent le comportement caractéristique d'une intoxication [7]. Les abeilles sont prises de tremblements, se réfugient sur les pétales ou tombent au sol et sont incapables de rejoindre la ruche. Dans les cas moins sévères, il n'y a pas d'effets spectaculaires mais les fonctions vitales peuvent être suffisamment perturbées pour entraîner d'autres effets à plus long terme (incapacité à butiner, à se nourrir, à communiquer, etc.).

L'imidaclopride peut être considéré comme très toxique pour les abeilles. En effet, la dose létale $50 \%$ (DL50) est de l'ordre de 4,10-9 grammes d'imidaclopride pour une abeille : (4 ng/abeille) [8-11]. Récemment, des travaux ont montré qu'en toxicité chronique, les doses pouvaient être encore plus basses et situées à partir de 0,01 ng/ abeille, ce qui correspond à une contamination de $0,1 \mu \mathrm{g} / \mathrm{kg}$ en masse relative d'imidaclopride dans la source de nourriture [12]. La toxicité peut également être évaluée chez l'abeille au niveau sub-létal, c'est-à-dire s'agissant des concentrations qui induisent des effets délétères sans provoquer de mortalité directe et immédiate. Par exemple l'activité de butinage est affectée, dès les premiers jours, avec une contamination de $3 \mu \mathrm{g} / \mathrm{kg}$ $[13,14]$. Il est à noter que la dégradation de l'imidaclopride dans les sols ou dans les plantes s'accompagne de la production de métabolites qui sont apparus aussi actifs que l'imidaclopride en toxicité sub-chronique [12].

Finalement, une telle situation nécessite de pouvoir décrire à l'échelle $\mathrm{du} \mu \mathrm{g} / \mathrm{kg}$, donc avec une très grande sensibilité, les quantités d'imidaclopride auxquelles les abeilles sont exposées lors de leur activité de butinage sur les grandes cultures traitées. Les techniques analytiques, à l'époque de la mise sur le marché de cet insecticide, ne possédaient pas une telle sensibilité. Aussi, nous avons développé de nouvelles méthodes ultrasensibles et parfaitement validées dans le cadre de notre expertise.

\section{Analyse}

En 1993, il n'était pas possible de détecter et de quantifier moins de $50 \mu \mathrm{g} / \mathrm{kg}$ d'imidaclopride dans un prélèvement de terrain $(20 \mu \mathrm{g} / \mathrm{kg}$ dans les cas les plus favorables) [15]. De plus, il n'y avait pas de méthodologie adaptée pour l'analyse des pollens. Un travail de recherches et de développements multiples a donc été nécessaire. Il concerne à la fois les performances de l'extraction, de la séparation et de la détection de la molécule recherchée. Nous avons mis au point des méthodologies analytiques pour l'étude des sols, des végétaux et des pollens en 1999-2000 [16-18]. De telles méthodes utilisent les capacités du couplage de la chromatographie en phase liquide pour la séparation, avec la spectrométrie de masse en tandem (HPLC/APCI/MS/MS) pour la détection. Aujourd'hui, la limite de détection (LOD) est de $0,1 \mu \mathrm{g} / \mathrm{kg}$ tandis que la limite de quantification (LOQ) est de $1 \mu \mathrm{g} / \mathrm{kg}$ [19]. Cela représente une amélioration de la sensibilité analytique supérieure à un facteur de 50 à 100 tandis que l'étude des pollens est désormais possible. 
L'application des nouveaux outils analytiques a permis de suivre l'imidaclopride pour de nombreux sites du territoire français. Ces sites diffèrent par leur composition géologique, leur pluviométrie, leur ensoleillement, le type de cultures, etc. De cette façon, nous avons pu obtenir une large description permettant d'aborder la contamination de manière assez aléatoire ; c'est un indicateur général de l'état des lieux de la situation française. Tous les échantillons ont été systématiquement comparés à des échantillons provenant de sites pratiquant l'agriculture biologique et pour lesquels aucune trace d'imidaclopride n'a été détectée.

\section{Résultats pour les sols}

La dose d'imidaclopride en traitement pour le tournesol est de $76 \mathrm{~g}$ par hectare. Ramené à $1 \mathrm{~kg}$ de terre, cela correspondrait au départ à $25 \mu \mathrm{g} /$ $\mathrm{kg}$. Les analyses de sols traités Gaucho ${ }^{\circledR}$ ont révélé une concentration moyenne de l'ordre de $12 \mu \mathrm{g} /$ $\mathrm{kg}$ en fin de culture (fig. 1). Un an plus tard, cette valeur moyenne est encore de $6 \mu \mathrm{g} / \mathrm{kg}$ en l'absence de nouveau traitement [20, 21]. Ces résultats illustrent la forte rémanence de l'imidaclopride dans les sols, ce qui le rend bio-disponible 1 à 2 années après le traitement initial [17, 20].

\section{Résultats pour les plantes}

Nous avons alors étudié le devenir de l'imidaclopride et de ses principaux métabolites lors de la croissance du tournesol; ceci dans les tiges, les feuilles et les capitules. Bien entendu, la teneur en imidaclopride diminue très rapidement dans le tournesol traité, au fur et à mesure de sa croissance. C'est au stade 59 [22], qui correspond à l'apparition du bouton floral, que le minimum est atteint. Une telle diminution pourrait laisser penser que la fleur ne contient plus de quantité pertinente d'imidaclopride à la floraison. Expérimentalement, ce n'est pas le cas, car un nouveau phénomène a été mis en évidence dès la formation du capitule. En effet, c'est à partir de ce stade

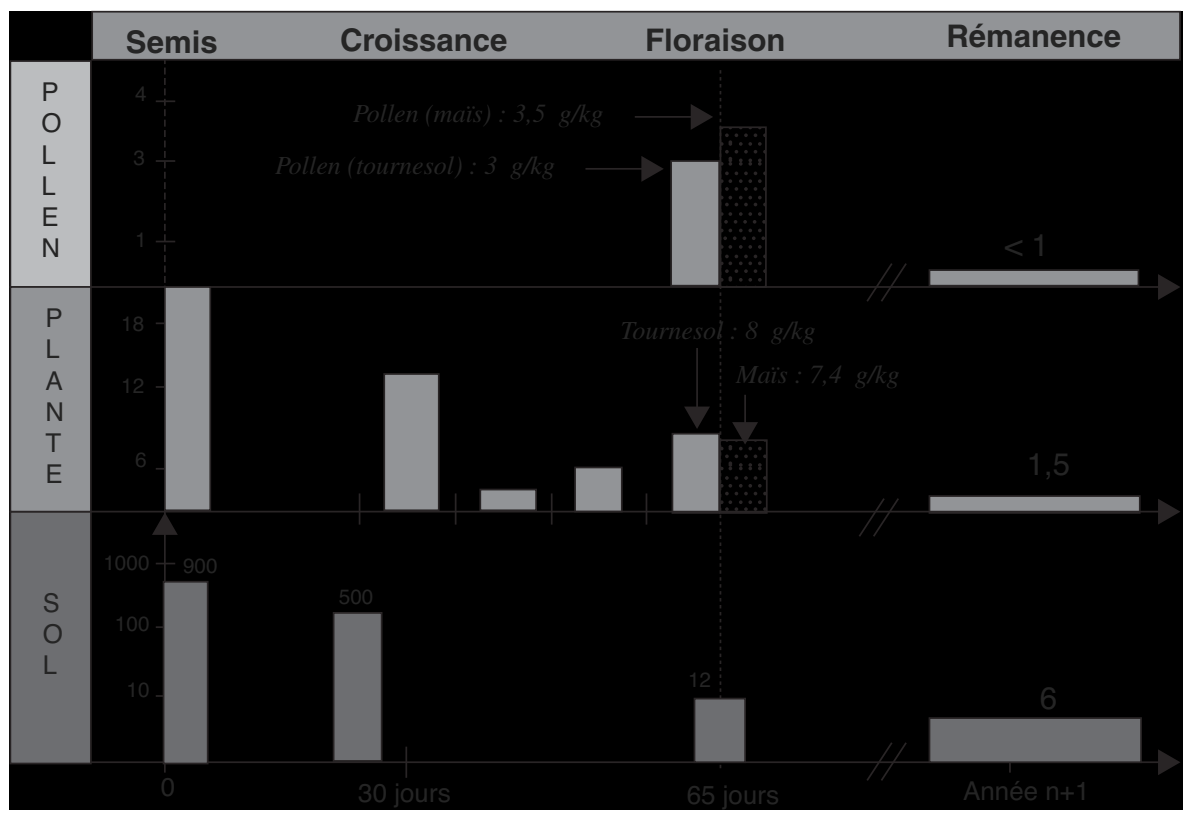

Figure 1. Quantités moyennes d'imidaclopride $(\mu \mathrm{g} / \mathrm{kg})$ dans chaque matrice en fonction du temps écoulé après le semis de la culture enrobée.

Mean quantities of imidacloprid $(\mu \mathrm{g} / \mathrm{kg})$ in each matrix as a function of time after seeding enrobed culture. 
que les quantités d'imidaclopride cessent de diminuer pour augmenter significativement, jusqu'à la pleine floraison (stades 61 et 65). Une illustration est donnée sur la figure 1. En fait, la valeur moyenne atteinte dans le capitule est de $8 \mu \mathrm{g} / \mathrm{kg}$ à la période de butinage $[19,20]$. Des analyses similaires, réalisées sur du maïs traité, ont montré que les concentrations moyennes dans les fleurs sont du même ordre de grandeur $(7,4 \mu \mathrm{g} / \mathrm{kg})$. Ainsi, lorsque ces deux espèces végétales sont traitées, elles révèlent des teneurs en imidaclopride assez élevées dans les parties supérieures de la plante et notamment dans les parties florales. Ceci peut s'expliquer par un métabolisme accru lors de la floraison et par une mobilisation importante des ressources de ces plantes qui sont d'importantes productrices de graines. De plus, ces cultures ont une forte capacité à recouvrer l'imidaclopride résiduel contenu dans le sol, à l'issue d'une culture traitée Gaucho ${ }^{\circledR}$ l'année précédente $[17,20]$.

\section{Résultats pour les pollens}

De par la contamination observée dans les fleurs, il n'est pas surprenant que les analyses des pollens de tournesol et de maïs aient révélé que les concentrations moyennes d'imidaclopride sont de l'ordre de $3 \mu \mathrm{g} / \mathrm{kg}[19,20]$. Ici, l'étude a porté aussi bien sur des pollens de fleurs que sur des pollens récoltés à l'entrée de la ruche, dits pollens de trappe. Dans le cas du maïs, la concentration moyenne de toxique dans les pollen de trappe est bien inférieure à celle mesurée dans les pollens de fleurs. Cela s'explique par la dilution liée à la dispersion du butinage et donc par le mélange de pollens provenant de maïs traités avec d'autres pollens de cultures non traitées. Dans ce cas, la moitié des pollens de trappe contient une concentration en imidaclopride supérieure à $0,1 \mu \mathrm{g} / \mathrm{kg}$.

\section{Conclusion}

Nous avons développé de nouvelles méthodes analytiques très performantes afin de déterminer la teneur en insecticide imidaclopride dans les sols, les végétaux et les pollens. La persistance de l'imidaclopride dans les sols a été mise en évidence plus d'un an, voire deux ans, après le traitement des cultures. Nos données de terrain corroborent celles des essais en laboratoire et confirment que le temps de demi-vie de l'imidaclopride dans les sols est élevé (3-6 mois) [23]. Par ailleurs, nous avons pu expliquer comment des plantes non-traitées comme le tournesol, le maïs ou les adventices (plantes non-semées) peuvent récupérer l'imidaclopride résiduel d'une culture traitée l'année précédente. Enfin, un nouveau phénomène d'augmentation de la teneur en toxique dans les fleurs de tournesol a été découvert. C'est à la pleine floraison que la quantité d'imidaclopride est maximale dans le capitule. De nouveau, ces résultats de terrain sont confirmés par d'autres études utilisant l'insecticide marqué au carbone radioactif [24]. S'agissant des pollens, qui représentent un intérêt majeur pour les abeilles, il est apparu qu'ils contiennent des doses significatives de toxique : de 0,1 à $3 \mu \mathrm{g} / \mathrm{kg}$ en moyenne. C'est justement la gamme de concentrations pour laquelle les premiers effets sub-létaux sur le butinage ou subchroniques ont été mis en évidence [12, 14]. Une telle situation permet d'évaluer les risques encourus par les abeilles face aux cultures de tournesol et de maïs traités, sachant que d'autres facteurs sont également à prendre en compte. En effet, la toxicité de tous les métabolites de l'imidaclopride $[25,26]$ ne doit pas être négligée [11, 12].

Ce dossier illustre combien les procédures d'autorisation de mise sur le marché de produits phytosanitaires doivent prendre en compte le comportement détaillé de ces molécules dans l'environnement. Le ministre de l'Agriculture et de la Pêche a provisoirement interdit la commercialisation et l'utilisation du Gaucho ${ }^{\circledR}$ sur tournesol en 1999 [27]. Cette décision, prise en application du principe de précaution, fut confirmée en 2001 au niveau du Conseil d'État et reconduite en 2003. À l'inverse, l'utilisation du Gaucho $^{\circledR}$ sur les cultures de maïs reste autorisée.

L'ensemble de ces études a montré que l'utilisation de cette nouvelle génération d'insecticide pourrait également avoir ses limites en matière de protection de l'environnement. Pour pallier aux risques de pollution due à la grande perte de produits actifs lors des pulvérisations, les récents insecticides ont vu leur toxicité accrue et leur 
mode d'application optimisé. L'efficacité de cette famille d'insecticides est susceptible de se révéler néfaste au regard de la persistance dans l'environnement et de la toxicité létale, sub-létale et chronique qu'elle peut engendrer. Ces toxiques, devenus très performants pour les insectes indésirables, le sont aussi pour les pollinisateurs lorsque les pollens ou nectars sont contaminés. De là, ils peuvent pénétrer dans la ruche et être une voie d'intoxication de la colonie entière. Ainsi, d'une certaine façon, le problème de l'accumulation des pesticides a été remplacé par celui de leur bio-disponibilité, c'est-à-dire par des quantités moindres mais où les matières actives sont plus toxiques et disponibles plus longtemps dans les écosystèmes.

\section{Remerciements}

Ces recherches ont été réalisées dans le cadre du Règlement CE 1221/97. Sont remerciés, pour leur participation respective aux présents travaux, les membres de l'équipe (E. Bengsch, S. Lecoublet et I. Moineau) et nos principaux collaborateurs (V. Charrier et C. Fléché).

\section{Références}

1. Moriya K, Shibuya K, Hattori J et al. Structural modification of the 6-chloropyridyl moiety in the imidacloprid skeleton: introduction of a five-membered heteroaromatic ring and the resulting insecticidal activity. Biosci Biotech Biochem 1992; 56: 364-5.

2. Bai D, Lummis SCR, Leicht W et al. Actions of imidacloprid and a related nitromethylene on cholinergic receptors of an identified insect motor neurone. Pestic Sci 1991; 33: 197-204.

3. Liu MY, Casida E. High affinity binding of 3H-imidacloprid in the insect acetylcholine receptor. Pestic Biochem Physiol 1993; 46: 40-6.

4. Leicht W. Imidacloprid - a chloronicotinyl insecticide. Pesticide Outlook 1993; 4: 17-21.

5. Yamamoto I, Yabuta G, Tomizawa M et al. Molecular Mechanism for selective toxicity of nicotinoids and neonicotinoids. J Pestic Sci 1995; 20: 33-40.

6. Buckingham S, Lapied B, Corronc $\mathrm{H}$ et al. Imidacloprid actions on nicotinic and mixed insect neuronal acetylcholine receptors. J Exp Biol 1997; 200: 2685-92.
7. Colin ME, Bonmatin JM. Enregistrement et analyse des anomalies de butinage constatées en 1999 en Vendée. In Rapport au ministère de l'Agriculture et de la Pêche, 2000.

8. Nauen R, Ebbinghaus-Kintscher U, Schmuck R. Toxicity and nicotinic acetylcholine receptor interaction of imidacloprid and its metabolites in Apis mellifera (Hymenoptera: Apidae). Pest Management Science 2001; 57: 577-86.

9. Schmuck R, Schöning R, Stork A et al. Risk posed to honeybees (Apis mellifera L, Hymenoptera) by an imidacloprid seed dressing of sunflowers. Pest Manag Sci 2001; 57: 225-38.

10. Suchail $\mathrm{S}$ et al. Characteristics of imidacloprid toxicity in two Apis Mellifera subspecies. Environ Toxicol Chem 2000; 19: 1901-5.

11. Suchail S, Guez D, Belzunces LP. Discrepancy between acute and chronic toxicity induced by imidacloprid and its metabolites in apis mellifera. Environ Toxicol Chem 2001; 20: 2482-6.

12. Guez D, Suchail S, Gauthier M et al. Contrasting effects of imidacloprid on habituation in 7 and 8 days old honeybees (Apis mellifera). Neurobiol Learn Mem 2001; 76: 183-91.

13. Pham-Delègue $\mathrm{MH}$, Cluzeau $\mathrm{S}$. Effets des produits phytosanitaires sur l'abeille. Incidence du traitement des semences de tournesol par Gaucho ${ }^{\circledR}$ sur les disparitions de butineuses. In Rapport final de synthèse au ministère de l'Agriculture et de la Pêche, 1999.

14. Colin ME, Bonmatin JM. Effets de très faibles concentrations d'imidaclopride et dérivés sur le butinage des abeilles en conditions semi-contrôlées. In Rapport au ministère de l'Agriculture et de la Pêche, 2000.

15. Placke FJ, Weber E. Method for determination of imidacloprid residues in plant materials. PflanzenschutzNachrichten Bayer 1993; 46: 2.

16. Bonmatin JM, Moineau I, Lecoublet $S$ et al. L'insecticide imidaclopride : bio-disponibilité dans les sols et les plantes, toxicité et risque pour les abeilles. Revue Française d'Apiculture $2000 ; 609: 360-1$.

17. Bonmatin JM, Moineau I, Lecoublet $S$ et al. Neurotoxiques systémiques : biodisponibilité, toxicité et risque pour les insectes pollinisateurs. Le cas de l'imidaclopride. Produits Phytosanitaires, Presses Universitaires de Reims, 2001, 175-81.

18. Bonmatin JM, Moineau I, Lecoublet S et al. Proceeding of the 3rd European Pesticide Residue Workshop. Pesticides in Food and drink, York, Central Science Laboratory Ed., 2000, 134.

19. Bonmatin JM, Moineau I, Charvet C et al. A LC/APCIMS/MS Method for analysis of imidacloprid in soils, in plants, and in pollens. Anal Chem 2003, 75, 2027-33. 
20. Bonmatin JM. Insecticide et pollinisateurs : une dérive de la chimie ? Sciences 2002; 2: 42-6.

21. Bonmatin JM, Moineau I, Charvet C et al. Behaviour of imidacloprid in fields. Toxicity for honey bees. Environmental Chemistry, Springer Ed., sous presse.

22. Lancashire PD, Bleiholder H. A uniform decimal code for growth stages of crops and weeds. Ann Appl Bio 1991; 119: 561-601.

23. Belzunces LP, Tasei JN. Impacts sur les dépeuplements de colonies d'abeilles et sur les miellées. In Rapport au ministère de l'Agriculture sur les effets des traitements de semences de tournesol au Gaucho ${ }^{\circledR}$ (imidacoprid), 1997.

24. Laurent F, Scalla R. Transport et métabolisme de l'imidaclopride chez le tournesol. $3^{\mathrm{e}}$ Programme Communau- taire pour l'apiculture. Années 1999-2000. In Rapport au ministère de l'Agriculture et de la Pêche, 2000.

25. Nauen R, Hungenberg H, Tollo B et al. Antifeedant effect, biological efficacy and high affinity binding of imidacloprid to acetylcholine receptors in Myzus persicae and Myzus nicotianae. Pest Management Science 1998; 53: 133-40.

26. Oliveira RS, Koskinen WC, Werdin NR et al. Sorption of imidacloprid and its metabolites on tropical soils. J Environ Sci Health (Br) 2000; 35: 39-49.

27. Journal officiel de la République française. Avis aux détenteurs et aux utilisateurs de semences de tournesol. Ministère de l'Agriculture et de la Pêche, 14 février 1999 , 2413. 